\title{
The potentially beneficial central nervous system activity profile of ivacaftor and its metabolites
}

\author{
Elena K. Schneider (10 ${ }^{1,2}$, Rachel M. McQuade ${ }^{3}$, Vincenzo C. Carbone ${ }^{4}$, \\ Felisa Reyes-Ortega ${ }^{5}$, John W. Wilson 6,7 , Brenda Button ${ }^{6,7}$, Ayame Saito ${ }^{3}$, \\ Daniel P. Poole ${ }^{3}$, Daniel Hoyer ${ }^{2,8,9}$, Jian Li ${ }^{10,11}$ and Tony Velkov ${ }^{1,2,11}$
}

\begin{abstract}
Affiliations: ${ }^{1}$ Drug Delivery, Disposition and Dynamics, Monash Institute of Pharmaceutical Sciences, Monash University, Parkville, Australia. ${ }^{2}$ Dept of Pharmacology and Therapeutics, School of Biomedical Sciences, Faculty of Medicine, Dentistry and Health Sciences, The University of Melbourne, Parkville, Australia. ${ }^{3}$ Drug Discovery Biology, Monash Institute of Pharmaceutical Sciences, Monash University, Parkville, Australia. ${ }^{4}$ Animal Science, AgResearch Limited, Palmerston North, New Zealand. ${ }^{5}$ Dept of Applied Physics, Sciences Faculty, University of Granada, Granada, Spain. ${ }^{6}$ Dept of Medicine, Monash University, The Alfred Hospital, Melbourne, Australia. ${ }^{7}$ Cystic Fibrosis Service, The Alfred Hospital, Melbourne, Australia. ${ }^{8}$ The Florey Institute of Neuroscience and Mental Health, The University of Melbourne, Parkville, Australia. ${ }^{9}$ Dept of Molecular Medicine, The Scripps Research Institute, La Jolla, CA, USA. ${ }^{10}$ Monash Biomedicine Discovery Institute, Dept of Microbiology, Monash University, Clayton, Australia. ${ }^{11}$ These two authors contributed equally to this work.
\end{abstract}

Correspondence: Tony Velkov, Dept of Pharmacology and Therapeutics, The University of Melbourne, Parkville, Victoria 3010, Australia. E-mail: tony.velkovdunimelb.edu.au

ABSTRACT Ivacaftor-lumacaftor and ivacaftor are two new breakthrough cystic fibrosis transmembrane conductance modulators.

The interactions of ivacaftor and its two metabolites hydroxymethylivacaftor (iva-M1) and ivacaftorcarboxylate (iva-M6) with neurotransmitter receptors were investigated in radioligand binding assays.

Ivacaftor displayed significant affinity to the 5-hydroxytryptamine (5-HT; serotonin) 5 - $\mathrm{HT}_{2 \mathrm{C}}$ receptor $(\mathrm{pK}=6.06 \pm 0.03), \beta_{3}$-adrenergic receptor $(\mathrm{pKi}=5.71 \pm 0.07), \delta$-opioid receptor $(\mathrm{p} K \mathrm{i}=5.59 \pm 0.06)$ and the dopamine transporter $(\mathrm{pKi}=5.50 \pm 0.20)$; iva-M1 displayed significant affinity to the $5-\mathrm{HT}_{2 \mathrm{C}}$ receptor $(\mathrm{pK}=5.81 \pm 0.04)$ and the muscarinic $\mathrm{M} 3$ receptor $(\mathrm{p} K \mathrm{i}=5.70 \pm 0.10)$; iva-M6 displayed significant affinity to the $5-\mathrm{HT}_{2 \mathrm{~A}}$ receptor $(\mathrm{pK \textrm {i }}=7.33 \pm 0.05)$. The in vivo central nervous system activity of ivacaftor $\left(40 \mathrm{mg} \cdot \mathrm{kg}^{-1}\right.$ intraperitoneally for 21 days) was assessed in a chronic mouse model of depression. In the forced swim test, the ivacaftor-treated group displayed decreased immobility $(52.8 \pm 7.6 \mathrm{~s})$, similarly to fluoxetine $(33.8 \pm 11.0 \mathrm{~s})$, and increased climbing/swimming activity $(181.5 \pm 9.2 \mathrm{~s})$. In the open field test, ivacaftor produced higher locomotor activity than the fluoxetine group, measured both as mean number of paw touches (ivacaftor $81.1 \pm 9.6$ versus fluoxetine $57.9 \pm 9.5$ ) and total distance travelled (ivacaftor $120.6 \pm 16.8 \mathrm{~cm}$ versus fluoxetine $84.5 \pm 16.0 \mathrm{~cm}$ ) in $600 \mathrm{~s}$. Treatment of 23 cystic fibrosis patients with ivacaftor-lumacaftor resulted in significant improvements in quality of life (including anxiety) in all five domains of the AweScoreCF questionnaire ( $\mathrm{p}=0.092-0.096)$.

Our findings suggest ivacaftor displays potential clinical anxiolytic and stimulating properties, and may have beneficial effects on mood.

@ERSpublications

The novel CFTR potentiator ivacaftor displays potential pre-clinical and clinical anxiolytic and antidepressant properties, which may have beneficial effects on mood and well-being in CF patients receiving ivacaftor or ivacaftor-lumacaftor therapy http://ow.ly/thK430iaigr

Cite this article as: Schneider EK, McQuade RM, Carbone VC, et al. The potentially beneficial central nervous system activity profile of ivacaftor and its metabolites. ERJ Open Res 2018; 4: 001272017 [https://doi.org/10.1183/23120541.00127-2017].

This article has supplementary material available from openres.ersjournals.com

The content of this work is @ the authors or their employers. Design and branding are @ERS 2018. This article is open access and distributed under the terms of the Creative Commons Attribution Non-Commercial Licence 4.0. 


\section{Introduction}

Cystic fibrosis (CF) is an autosomal recessive genetic disease with an incidence of 1:2500-3500 in Caucasian populations [1]. CF affects the exocrine mucus glands of the lung, liver, pancreas and intestine, causing progressive multisystem failure, such as loss of lung function or pancreatic insufficiency [1]. CF results from mutations of the CF transmembrane conductance regulator (CFTR) gene that affect chloride ion channel function; this in turn leads to dysregulation of epithelial fluid transport in various organs, more specifically in the lung, pancreas and other organs. Complications include thickened mucus in the airways associated with frequent respiratory infections, and pancreatic insufficiency giving rise to malnutrition and diabetes. These conditions lead to chronic disease status, poorer quality of life and reduced quality of life. Despite significant advances in diagnosis and therapy, CF remains one of the most challenging diseases to manage. Although a slew of medications such as antibiotic, anti-inflammatory and mycolytic agents have increased the life expectancy of CF patients, quality of life is still significantly impaired [2]. It is recognised that with the inevitable progression of the disease, patients require more intensive therapies, that themselves may impact on quality of life [3].

A number of drugs act in the central nervous system (CNS) and peripheral nervous system as well as in many other systems/organs by targeting ligand-gated or receptor-gated ion channels, receptors or transporters. The development of selective modulators of CFTR has changed the spectrum of the management of CF in patients. Ivacaftor $150 \mathrm{mg}$ once every $12 \mathrm{~h}$ was the first clinically approved CFTR modulator with clinical efficacy showing a significant improvement in the lung function of CF patients bearing the specific mutation G551D-CFTR, found in $4-5 \%$ of CF patients [4]. Ivacaftor was later approved for other gating mutations, e.g. G1244E, G1349D, G178R, G551S, S1251N, S1255P, S549N or S549R [5]. The CFTR potentiator activity of ivacaftor results in increased CFTR channel opening probability to enhance chloride efflux [6]. Ivacaftor is extensively metabolised by cytochrome P450 enzymes, mainly to an active metabolite hydroxymethylivacaftor (iva-M1) and to an inactive form ivacaftorcarboxylate (iva-M6) [7]. The F508del mutation is another common mutation of CFTR, which produces a misfolded and defective protein that is present at the apical epithelial membrane, but is inactive [8]. Ivacaftor is ineffective on the F508del mutated form of CFTR. Ivacaftor-lumacaftor is a novel combination treatment that combines the potentiator function of ivacaftor with the CFTR corrector function of lumacaftor, the latter improving trafficking of the more functional CFTR to the cell surface [9]. This novel combination strategy effectively expands the therapeutic window to the broader CF patient collective [9]. Although ivacaftor and ivacaftor-lumacaftor have only recently entered clinical use, various independent laboratories have reported unexpected off-target effects ranging from the direct antimicrobial activity of ivacaftor [10], synergistic antimicrobial activity of ivacaftor-lumacaftor in combination with polymyxin B [11] to smooth muscle relaxation activity of ivacaftor in CF patients [12].

Fundamentally, ivacaftor is an ion channel modulator and as such may impact on other ion channels, particularly in the CNS wherein ligand-gated ion channels mediate neurotransmission processes. In the present study, we investigated the receptor pharmacology and CNS activity of ivacaftor and its main metabolites, i.e. iva-M1 and iva-M6. Interactions of ivacaftor, iva-M1 and iva-M6 with a range of neurotransmitter receptors and transporters were investigated in radioligand binding assays together with molecular docking techniques to the 5-hydroxytryptamine (5-HT; serotonin) $5-\mathrm{HT}_{2 \mathrm{~A}}$ and $5-\mathrm{HT}_{2 \mathrm{C}}$ receptors. The $5-\mathrm{HT}_{2 \mathrm{C}}$ receptor is the target of a number of antidepressants, most prominently agomelatine [13-15]. Agomelatine is a 5- $\mathrm{HT}_{2 \mathrm{C}}$ receptor antagonist and melatonin 1 and 2 receptor agonist, which is effective in major depression and general anxiety disorders $[13,14]$. The $5-\mathrm{HT}_{2 \mathrm{C}}$ receptor is also the target of drugs acting on eating disorders/obesity, e.g. lorcaserin [16-18], or schizophrenia, e.g. vabicaserin [19]; these compounds are typically $5-\mathrm{HT}_{2 \mathrm{C}}$ receptor agonists, in contrast to agomelatine which is an antagonist [20]. The 5- $\mathrm{HT}_{2 \mathrm{C}}$ receptor has been linked to depression, anxiety, panic, suicide, schizophrenia, epilepsy, obesity and addiction based on behavioural effects observed in transgenic animals [20-25], using selective agonists [13-17, 26-32], genome-wide association studies [33, 34] or clinical studies with new drug candidates [18, 19]. The field is not without controversies, and both agonists and

This article has supplementary material available from openres.ersjournals.com

Received: Oct 112017 | Accepted after revision: Jan 292018

Conflict of interest: None declared.

Support statement: J. Li and T. Velkov are supported by the National Institute of Allergy and Infectious Diseases of the National Institutes of Health (R01 AI111965). J. Li is an Australian National Health and Medical Research Council (NHMRC) Senior Research Fellow and T. Velkov is an Australian NHMRC Industry Career Development Level 2 Research Fellow. D. Hoyer is supported by the NHMRC. E.K. Schneider is an appointed Young Ambassador for the American Society of Microbiology and acknowledges support from the Australian Postgraduate Award. Funding information for this article has been deposited with the Crossref Funder Registry. 
antagonists may produce similar effects on, for example, depression readouts $[15,35]$. However, it is clear that agonists will decrease food intake in contrast to antagonists and it appears that antagonists seem to increase dopaminergic function, which may explain the increase in locomotor activity that can be observed with such compounds, whereas agonists may reduce locomotor activity [13, 36, 37].

The drugs were profiled for neurotransmitter receptor and transporter binding affinity using radioligand binding assays. The in vivo antidepressant potential of ivacaftor was assessed using the forced swim test in a chronic non-CF mouse model of depression. Potential anxiolytic activity and effects on locomotor activity were evaluated in an open field test measuring spontaneous locomotor activity and exploration [38]. The M3 muscarinic receptor activity of ivacaftor and its metabolites was also assessed using contractile activity of the isolated colon as a functional readout. Furthermore, the effect of ivacaftor-lumacaftor therapy on well-being and mental state in $23 \mathrm{CF}$ patients was assessed via the AweScoreCF questionnaire. Our novel findings suggest that ivacaftor possesses unique off-target effects which translate into potential anxiolytic and stimulating properties that may be beneficial for alleviating mental health symptoms that commonly occur in eligible CF patients.

\section{Methods}

\section{Primary radioligand binding assays}

Primary radioligand binding assays were performed as described by Rотн [39]. Please refer to the supplementary material for a more detailed description.

Molecular modelling of the ivacaftor-5-HT $2 C$ and iva-M6-5-HT $2 A$ receptor complexes

Please refer to the supplementary material.

Animals

Please refer to the supplementary material.

Forced swim test

Ivacaftor was injected intraperitoneally at a dose of $40 \mathrm{mg} \cdot \mathrm{kg}^{-1}$ once daily (between 9 and 11 a.m.) for 21 days. The control, the selective serotonin reuptake inhibitor fluoxetine, was administered at a dose of $10.2 \mathrm{mg} \cdot \mathrm{kg}^{-1}$ i.p. once daily (between 9 and 11 a.m.) for 21 days. Ketamine was injected subcutaneously at a dose of $10 \mathrm{mg} \cdot \mathrm{kg}^{-1} 1 \mathrm{~h}$ prior to the testing. On the day of the forced swim test (day 21), the mice received the treatment $60 \mathrm{~min}$ before the swim test.

The forced swim test was performed as described by Ponsolt et al. [40]. Briefly, experiments were performed in a quiet and slightly dimmed experimental room (light intensity $\sim 80$ lux). Each mouse was placed into a transparent glass cylinder (diameter $20 \mathrm{~cm}$, height $40 \mathrm{~cm}$ ) filled with warm water $\left(23 \pm 1.0^{\circ} \mathrm{C}\right.$ ) and allowed to swim for 6 min while video recorded (PanLab Smart 3.0; Harvard Apparatus, Holliston, MA, USA). Care was taken to ensure that the level and the depth of water did not allow the mouse to escape or to support itself from the bottom of the cylinder. The video recordings of the groups were analysed by an experimenter blinded to the treatment groups. The cumulative duration of immobility and swimming/climbing during the last 4 min of each trial was measured using a stop watch.

\section{Assessment of spontaneous locomotor activity}

The open field test was performed 1 day after the forced swim test trials. Spontaneous locomotor activity was assessed using a computerised iPad-based platform serving as a floor $(21 \times 13.5 \mathrm{~cm})$ of a cage $15.5 \mathrm{~cm}$ in height. The system and the software recording the number of paw steps and the distance between them was purchased from Neurolytical (Ann Arbor, MI, USA). The recordings were performed in a quiet room and dim light conditions (10 lux). The mice were allowed to adapt in the test environment for at least 10 min before the start of the experiment.

\section{Statistical analysis of the animal models}

Please refer to the supplementary material.

Force contraction experiment

Please refer to the supplementary material.

\section{AweScoreCF patient questionnaire}

23 adult CF patients study participants (informed consent; study protocol ClinicalTrials.gov identifier NCT02717650 was approved by the local ethics committee) completed the AweScoreCF questionnaire (The Alfred Hospital, Melbourne, Australia) 1 month before initiating ivacaftor-lumacaftor therapy and again following 3 months of treatment. All 23 patients were clinically stable and did not receive 
psychotropic medication. The AweScoreCF is a questionnaire developed by ButTon et al. [41] to evaluate patients' own perception of the effects of ivacaftor-lumacaftor on their health and mental state. The questionnaire consists of five domains, with each domain consisting two questions: CF clinical parameters (sputum and coughing), physical fitness (energy and exercise participation), physique (appetite and weight), psychological parameters (mood and anxiety) and general well-being (duration and quality of sleep and general health). Statistical analysis was performed using Prism (GraphPad, La Jolla, CA, USA). The values are presented as the mean \pm SEM and differences were considered to be statistically significant at $\mathrm{p}<0.05$. The individual scores were analysed in a paired t-test using ANOVA.

\section{Results}

\section{Neurotransmitter receptor and transporter radioligand binding assays}

Initially, we performed a broad neurotransmitter receptor screen (primary neuroreceptor binding screen) using a single concentration $(10 \mu \mathrm{M})$ of ivacaftor, its metabolites iva-M1 and iva-M6, and the CFTR correctors tezacaftor and lumacaftor (supplementary table S1). The respective radioligands for each receptor or transporter and cell lines, cell media or buffers are documented in supplementary table S2 [39]. Ivacaftor and iva-M1 showed significant displacement of the radioligand from the $5-\mathrm{HT}_{2 \mathrm{C}}$ receptor (84.8\% and $78.2 \%$, respectively). Ivacaftor, iva-M1 and iva-M6 showed significant displacement of the radioligands to the $\alpha_{1 \mathrm{~A}}$-adrenergic receptor $(58.3 \%, 79.9 \%$ and $92.3 \%$, respectively) and to the serotonin transporter $(61.1 \%, 56.7 \%$ and $50.9 \%$, respectively). Furthermore, ivacaftor displayed significant displacement of the $\beta_{3}$-adrenergic receptor $(75.9 \%), \delta$-opioid receptor $(50.8 \%)$ and dopamine transporter binding (55.2\%). Tezacaftor and lumacaftor showed significant displacement of the radioligands from the histamine $\mathrm{H}_{1}$ receptor $(53.3 \%$ and $71.2 \%$, respectively) and to the peripheral benzodiazepine receptor (63.7\% and $82.9 \%$, respectively). Secondly, we obtained secondary radioligand concentration-response curves on the neurotransmitter receptors and transporters that showed significant displacement, where supplementary table S3 documents the inhibition constant $(K \mathrm{i})$ obtained from nonlinear regression of the radioligand competition curves. Ivacaftor showed high-to-moderate affinity for the 5- $\mathrm{HT}_{2 \mathrm{C}}$ receptor $\left(K \mathrm{i}=866 \mathrm{nM} ; \mathrm{pKi}=6.06 \pm 0.03\right.$ ) (figure 1) and muscarinic $\mathrm{M}_{1}$ receptor $(K \mathrm{i}=1546 \mathrm{nM} ; \mathrm{pKi}=5.81 \pm 0.04)$ (supplementary figure S1). Ivacaftor displayed moderate affinity for the $\beta_{3}$-adrenergic receptor $(K \mathrm{i}=1934 \mathrm{nM} ; \mathrm{p} K \mathrm{i}=5.71 \pm 0.07)$, dopamine transporter $(K \mathrm{i}=2935 \mathrm{nM} ; \mathrm{p} K \mathrm{i}=5.50 \pm 0.20)$ and $\delta$-opioid receptor $(K \mathrm{i}=2589 \mathrm{nM} ; \mathrm{p} K \mathrm{i}=5.59 \pm 0.06)$ (supplementary figure S1). Interestingly, iva-M1 displayed high-to-moderate affinity to the muscarinic $\mathrm{M} 3$ receptor $(K \mathrm{i}=1832 \mathrm{nM} ; \mathrm{pKi}=5.70 \pm 0.10)$ and the $5-\mathrm{HT}_{2 \mathrm{C}}$ receptor $(K \mathrm{i}=1546 \mathrm{nM} ; \mathrm{pK} \mathrm{i}=5.81 \pm 0.04)$ (supplementary figure $\mathrm{S} 2$ ). iva-M6 showed high affinity for the $5-\mathrm{HT}_{2 \mathrm{~A}}$ receptor $(K \mathrm{i}=47 \mathrm{nM} ; \mathrm{pKi}=7.33 \pm 0.05)$ (figure 1 and supplementary figure $\mathrm{S} 3$ ). Ivacaftor, iva-M1 and iva-M6 displayed low-affinity binding to the $\alpha_{1 \mathrm{~A}}$-adrenergic receptor and the serotonin transporter $\left(K_{\mathrm{i}}>10 \mu \mathrm{M}\right)$.
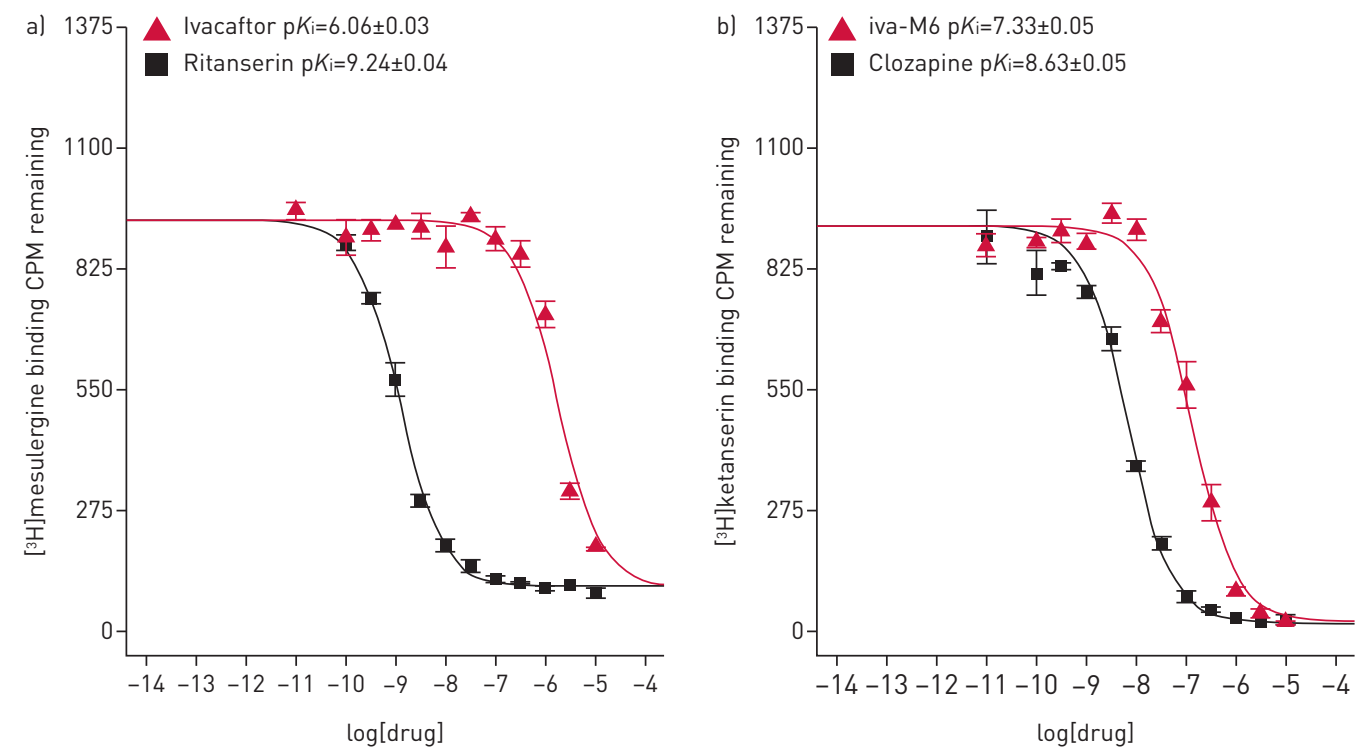

FIGURE 1 a) Secondary binding data for ivacaftor. $\left[{ }^{3} \mathrm{H}\right]$ mesulergine displacement data $(\mathrm{Ki}=866 \mathrm{nM})$ binding to the 5-hydroxytryptamine (5- $\mathrm{HT}$; serotonin) $5-\mathrm{HT}_{2 \mathrm{C}}$ receptor. The reference compound is ritanserin $\left(K_{i}=0.57 \mathrm{nM}\right)$. b) Secondary binding data for ivacaftorcarboxylate (iva-M6). [ $\left.{ }^{3} \mathrm{H}\right]$ ketanserin displacement data $\left(K_{i}=147 \mathrm{nM}\right)$ binding to the $5-\mathrm{HT}_{2 \mathrm{~A}}$ receptor. The reference compound is clozapine $\left(K_{i}=2.4 \mathrm{nM}\right)$. CPM: counts per minute. 
Tezacaftor (supplementary figure S4) and lumacaftor (supplementary figure S5) displayed moderate affinity to the $\alpha_{1 \mathrm{D}}$-adrenergic receptor $(K \mathrm{i}=5451 \mathrm{nM} ; \mathrm{p} K \mathrm{i}=5.26 \pm 0.08$ and $K \mathrm{i}=4838 \mathrm{nM} ; \mathrm{p} K \mathrm{i}=5.32 \pm 0.09$, respectively) and the peripheral benzodiazepine receptor $(K \mathrm{i}=1015 \mathrm{nM} ; \mathrm{p} K \mathrm{i}=5.99 \pm 0.07$ and $K \mathrm{i}=79 \mathrm{nM} ; \mathrm{p} K \mathrm{i}=7.1 \pm 0.07$, respectively). Lumacaftor displayed binding to the $\alpha_{1 \mathrm{~B}}$-adrenergic receptor, albeit with low affinity $(\mathrm{Ki}>10 \mu \mathrm{M})$.

Molecular modelling of the ivacaftor-5-HT $2 \mathrm{C}$ and iva-M6-5-HT $2 \mathrm{~A}$ receptor complexes Ivacaftor and iva-M6 showed significant affinity for the $5-\mathrm{HT}_{2 \mathrm{C}}$ and $5-\mathrm{HT}_{2 \mathrm{~A}}$ receptors, respectively. Intuitively, we modelled ivacaftor in complex with the $5-\mathrm{HT}_{2 \mathrm{C}}$ and $5-\mathrm{HT}_{2 \mathrm{~A}}$ receptors, and the top-ranking binding poses were examined (figure 2). The models described fall within reasonable confidence values for a valid "modelled" structure. The receptor binding domains for each ligand in both receptors are primarily created via interactions with extracellular loop domains (in particular EXL2) and transmembrane domain helices designated TM3, TM5, TM6 and TM7. Notably, large differences were observed for EXL2 (figure 2) and the size of the transmembrane helices that help position the various ligands for these receptors. In $5-\mathrm{HT}_{2 \mathrm{C}}$ and $5-\mathrm{HT}_{2 \mathrm{~A}}$ docking poses, the quinolone and hydroxyphenyl moieties nearly superimpose, differentiated mainly by the large number of hydrogen-bond interactions carried out by the carboxylate in the $5-\mathrm{HT}_{2 \mathrm{~A}}$ complex with the hydroxyl of Tyr370 (TM7; $3.1 \AA$ ), the amine of Trp367 (TM7; $3.0 \AA$ ) and the hydroxyl of Ser131 (TM2; $2.5 \AA)$. The hydroxyl on the phenol ring also forms a hydrogen-bond contact with the side chain hydroxyl of Tyr370 (TM7; $3.1 \AA$ ), while the carbonyl of iva-M6 forms van der Waals contacts with the carboxylate of Asp155 (TM3; $3.4 \AA$ ). Similar interactions are seen between identical moieties with Tyr358 in the 5- $\mathrm{HT}_{2 \mathrm{C}}$-ivacaftor complex (TM7; $2.7 \AA$ ) and Asp134 (TM3; $3.1 \AA$ ), while the hydroxyl of Ser110 (TM2; $2.9 \AA$ ) makes an additional hydrogen-bond contact with the hydroxyl on the phenol ring of ivacaftor. A hydrophobic cavity formed by TM3 (Val156 of 5- $\mathrm{HT}_{2 \mathrm{~A}}$ and Val135 of 5-HT $\mathrm{HC}_{2 \mathrm{C}}$ ), TM5 (Phe234 and Val235 of 5- $\mathrm{HT}_{2 \mathrm{~A}}$ and Phe214 and Val215 of 5- $\mathrm{HT}_{2 \mathrm{C}}$ ) and TM6 (Phe339 and Phe340 of $5-\mathrm{HT}_{2 \mathrm{~A}}$ and Phe327 of $5-\mathrm{HT}_{2 \mathrm{C}}$ ) mediates and stabilises extensive contact with the quinolone ring. Additional hydrophobic contacts occur via the tert-butyl moieties and the phenol ring, where in $5-\mathrm{HT}_{2 \mathrm{~A}}$ the phenol ring partially stacks Val366 on TM7, and makes hydrophobic contacts with Leu228 (EXL2), Leu362 (TM7), Trp151 and Ile152 (TM3), and Thr134 and Ile135 (TM2). In 5-HT 2 , ivacaftor makes no pertinent contact with EXL2 or TM2; all other interactions with hydrophobic tert-butyl moieties and phenol ring remain identical with corresponding residues Val354, Leu350, Trp130 and Ile131.

\section{Forced swim test}

As ivacaftor and its metabolites bind to the 5- $\mathrm{HT}_{2 \mathrm{~A}}$ receptor, 5- $\mathrm{HT}_{2 \mathrm{C}}$ receptor, $\delta$-opioid receptor, serotonin transporter and dopamine transporter, which are all involved in mood behaviour, we examined the CNS activity of ivacaftor using the forced swim test, the gold standard non-CF murine model for testing antidepressant properties (figure 3) [42]. The forced swim test evaluates the activity of a compound to increase swimming and climbing and to decrease immobility compared with vehicle. The forced swim
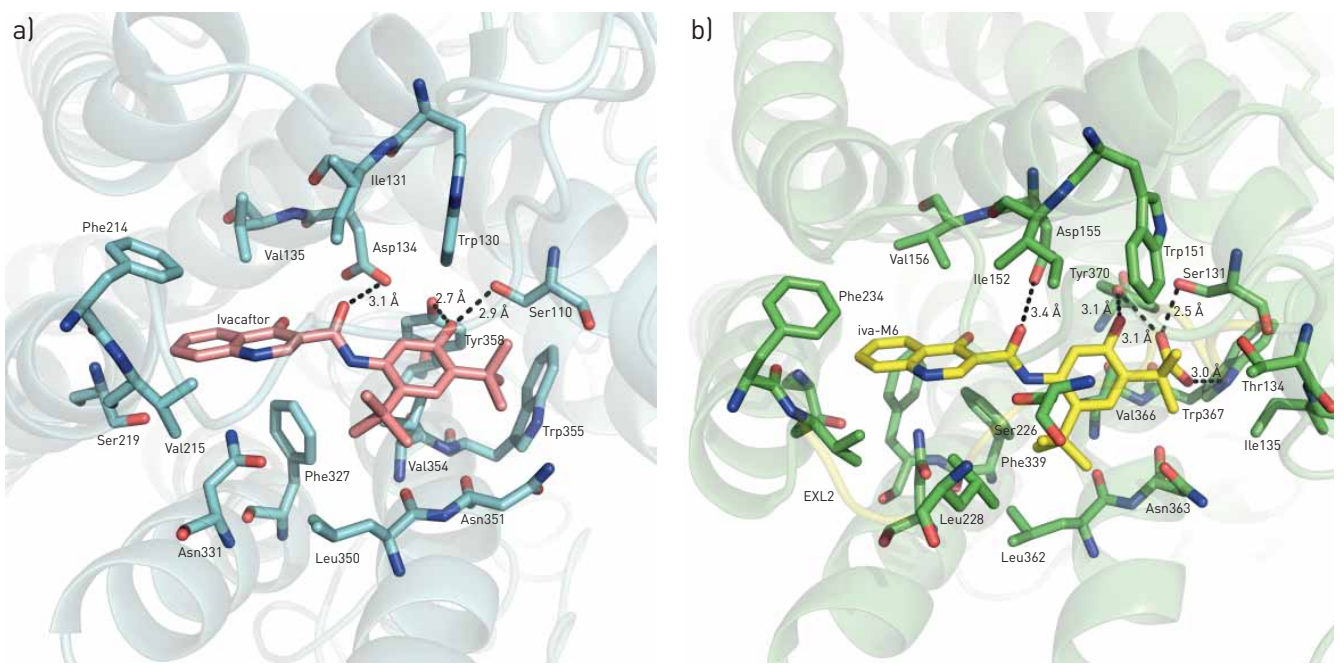

FIGURE 2 Molecular docking models of a) ivacaftor bound to the 5-hydroxytryptamine (5-HT; serotonin) $5-\mathrm{HT}_{2 \mathrm{C}}$ receptor and b) ivacaftorcarboxylate (iva-M6) bound to the $5-\mathrm{HT}_{2 \mathrm{~A}}$ receptor. Ivacaftor, iva-M6 and residues within the receptor cavity are shown in ball-and-stick representation. The $5-\mathrm{HT}_{2 \mathrm{C}}$ and $5-\mathrm{HT}_{2 \mathrm{~A}}$ receptors are shown in cartoon ribbon representation. The labelled side chains within the binding cavity represent contacts within $4 \AA$ of the docked molecules. 

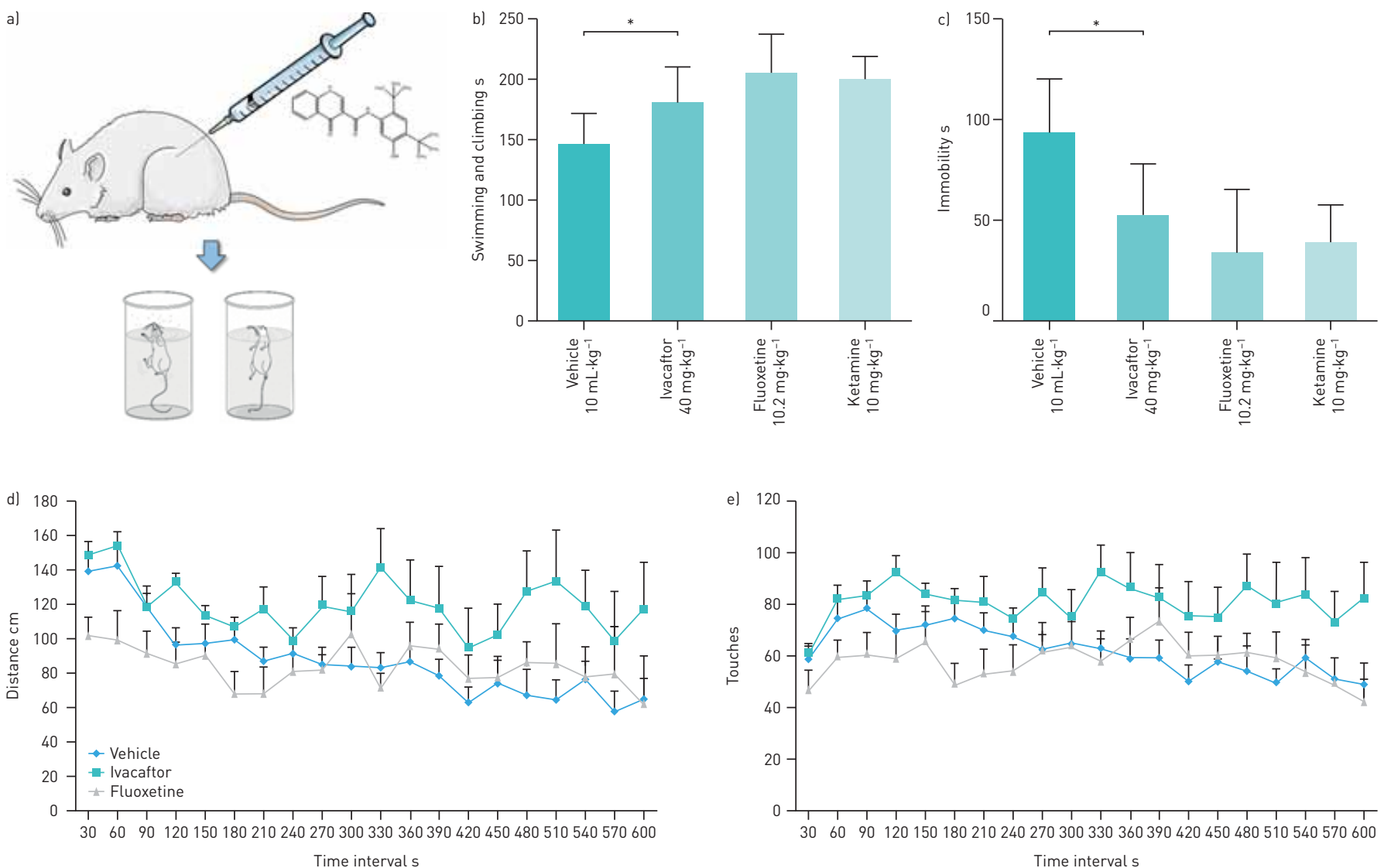

FIGURE 3 Results of the murine forced swim test and spontaneous locomotor activity tests for ivacaftor, fluoxetine and ketamine. al Schematic diagram depicting the forced swim test. b) Effect of ivacaftor treatment ( $40 \mathrm{mg} \cdot \mathrm{kg}^{-1}$ i.p. for 21 days) on swimming and climbing in the forced swim test $(\mathrm{n}=10)$. Fluoxetine $\left(10.2 \mathrm{mg} \cdot \mathrm{kg}^{-1}\right.$ i.p. for 21 days) and ketamine $\left(10 \mathrm{mg} \cdot \mathrm{kg}^{-1}\right.$ s.c.) were used as the comparators. c) Effect of ivacaftor, fluoxetine and ketamine on immobility in the forced swim test $(n=10) . *$ : $p<0.05$. d) Effect of ivacaftor and fluoxetine on spontaneous locomotor activity in the open field test measuring the distance $(\mathrm{cm})$ travelled for $10 \mathrm{~min}(\mathrm{n}=10)$. e) Effect of ivacaftor, fluoxetine and ketamine on spontaneous locomotor activity in the open field test measuring the number of touches for $10 \mathrm{~min}(\mathrm{n}=10)$.

test is highly reliable and has strong predictive validity for antidepressant compounds [40, 43]. Mice treated with vehicle showed baseline immobility for $93.7 \pm 8.2 \mathrm{~s}$ (figure 3c). Mice treated with a clinically relevant ivacaftor dose $\left(40 \mathrm{mg} \cdot \mathrm{kg}^{-1}\right)$ [7] showed decreased immobility with an average immobility period of $52.8 \pm 7.6 \mathrm{~s}$ (figure $3 \mathrm{~b}$ and $\mathrm{c}$ ). Similarly, the mice treated with ketamine $\left(10 \mathrm{mg} \cdot \mathrm{kg}^{-1}\right)$ or fluoxetine $\left(10.2 \mathrm{mg} \cdot \mathrm{kg}^{-1}\right)$ showed decreased immobility of $39.2 \pm 10.5$ and $33.8 \pm 11.0 \mathrm{~s}$, respectively. In addition, mice treated with ivacaftor, fluoxetine and ketamine showed increased swimming and climbing activities (ivacaftor $181.5 \pm 9.2 \mathrm{~s}$, fluoxetine $206.2 \pm 11.0 \mathrm{~s}$ and ketamine $200.8 \pm 8.1 \mathrm{~s}$ ) compared with vehicle $(146 \pm 8.2 \mathrm{~s})$ (figure $3 \mathrm{c}$ ).

\section{Open field test}

The open field test is a classic approach in which the novel environment concurrently evokes both anxiety and exploration [44-46]. The spontaneous locomotor activity of ivacaftor, fluoxetine and ketamine was assessed in mice with the open field test, which uses the number of paw touches and the total distance travelled as end-points (figure $3 \mathrm{~d}$ and e) [38]. The mice treated with ivacaftor $\left(40 \mathrm{mg} \cdot \mathrm{kg}^{-1}\right)$ showed higher locomotor activity $(120.6 \pm 16.8 \mathrm{~cm}$ distance travelled; $81.1 \pm 9.6$ paw touches) than the vehicle group $\left(88.4 \pm 10.5 \mathrm{~cm}\right.$ distance travelled; $62.4 \pm 7.2$ paw touches) and fluoxetine $\left(10.2 \mathrm{mg} \cdot \mathrm{kg}^{-1}\right)(84.5 \pm 16.0 \mathrm{~cm}$ distance travelled; $57.9 \pm 9.5$ paw touches).

\section{AweScoreCF questionnaire}

We assessed the general improvement in quality of life and mental health (measured as the individual perception of each patient) in 23 adult CF patients before commencing and after 3 months of standard ivacaftor-lumacaftor therapy using the AweScoreCF questionnaire (figure 4) [41]. All domain changes were in favour of improved quality of life. Changes were statistically significant: anxiety (mean of differences (MOD) 0.91 points), sleep (MOD 1.87 points), weight (MOD 1.44 points), cough (MOD 1.36 

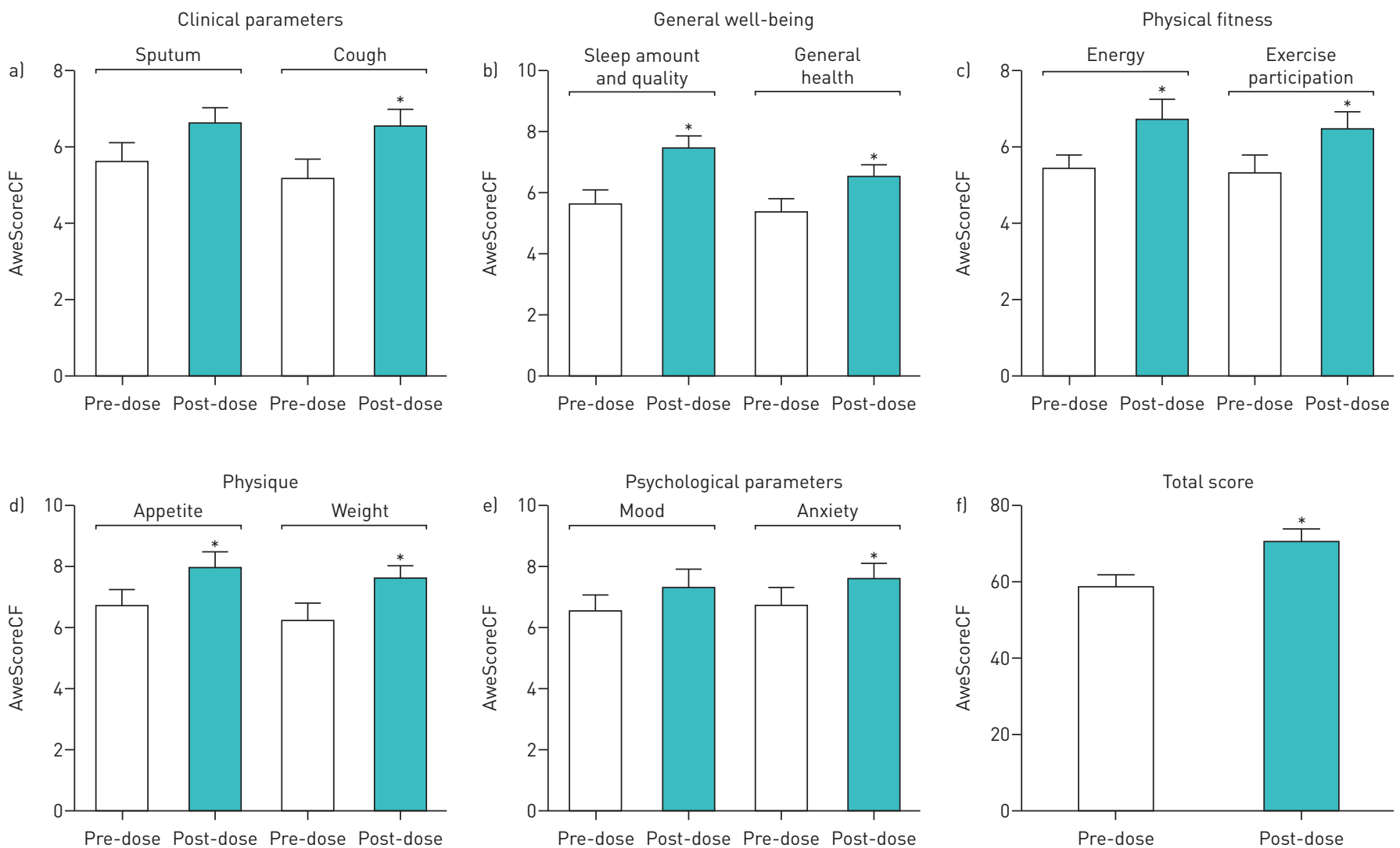

FIGURE 4 Results of the AweScoreCF questionnaire completed by 23 adult cystic fibrosis (CF) patients 1 month before and 3 months after initiating ivacaftor-lumacaftor therapy: al clinical parameters, b) general well-being, c) physical fitness, d) physique, e) psychological parameters and f) total score. Data are presented as mean \pm SEM of a paired t-test. *: $p<0.05$. In eight out of 10 subdomains changes resulted in statistically significant improvement of quality of life and well-being of the 23 adult CF patients; a positive trend was observed in the subdomains sputum and mood.

points), appetite (MOD 1.32 points), energy (MOD 1.28 points), general health and well-being (MOD 1.17 points), exercise participation (MOD 1.13 points), and total improvement in well-being (MOD 11.59 points), whereas amount of sputum (MOD 0.96 points) and mood (MOD 0.86 points) showed trends for improvement $(\mathrm{p}=0.092-0.096)$.

\section{Discussion}

The goal of this study was to identify off-target actions of ivacaftor and ivacaftor-lumacaftor therapy by investigating the receptor pharmacology of the active ingredients ivacaftor and its major metabolites, i.e. iva-M1 and iva-M6, and lumacaftor. The clinical benefit (improved lung function, weight and exacerbation frequency) and safety profiles of ivacaftor and lumacaftor, reported in the TRAFFIC and TRANSPORT studies [9], are reassuring; however, WAINWRIGHT et al. [9] also reported an increase in respiratory adverse events including dyspnoea and chest tightness compared with placebo. Additionally, various independent laboratories have previously reported beneficial and unfavourable off-target effects of ivacaftor. ADAM et al. [12] have reported the rapid and relaxing effects of ivacaftor on smooth muscle in human airway distensibility and vascular tone. Reznikov et al. [10] showed that ivacaftor displayed antistaphylococcal activity in vitro. SCHNEIDER et al. [11] reported synergistic antibacterial activity between polymyxin B in combination with ivacaftor and ivacaftor-lumacaftor combination against polymyxin-resistant Pseudomonas aeruginosa isolates. Furthermore, evidence has emerged from two independent laboratories suggesting that prolonged exposure to ivacaftor counteracts the corrector function of lumacaftor by destabilising the lumacaftor-rescued mature glycoform of F508del-CFTR [47-49]. The in vitro studies by CHOLON et al. [47] suggested that chronic administration of ivacaftor caused a dose-dependent reversal of lumacaftor-mediated CFTR correction in homozygous F508del human primary bronchial epithelial cells. These findings demonstrate that chronic treatment with CFTR potentiators and correctors may have unexpected effects that cannot be predicted from short-term studies. Therefore, it is essential that we gain further knowledge of the interaction and interference between CFTR potentiators and CFTR correctors. 
The data from the primary and secondary binding screen for ivacaftor, iva-M1 and iva-M6 clearly show that ivacaftor and iva-M1 display a high affinity to the $5-\mathrm{HT}_{2 \mathrm{C}}$ receptor, and iva-M6 showed very high affinity to the 5- $\mathrm{HT}_{2 \mathrm{~A}}$ receptor (supplementary tables $\mathrm{S} 1$ and $\mathrm{S} 3$, and figure 1). Although further studies have yet to prove the implications of these findings, various benefits may exist for widespread binding at serotonin receptors in addition to the serotonin transporter interaction ( $\mathrm{K} \geqslant 10 \mu \mathrm{M}$ for ivacaftor, iva-M1 and iva-M6). We have shown that ivacaftor is a multifaceted drug with multimodal activity. Although these pre-clinical findings clearly indicate ivacaftor produces an antidepressant/anxiolytic-like profile, behavioural consequences of the chronic administration of ivacaftor have not yet been described. Serotonin receptors and ion channels, primarily sodium, potassium, calcium and chloride channels, have been associated with many neuronal processes, and are currently being targeted and/or pursued as potential targets for the treatment of depression [50]. Antidepressants reduce immobility time in the forced swim test by increasing the swimming/climbing time [51]. It is known that drugs affecting noradrenergic neurotransmission, i.e. imipramine, increase the climbing tendencies, whereas drugs affecting the serotoninergic neurotransmission, i.e. fluoxetine, increase the swimming behaviour [51]. Animal models are crucial tools for investigating the aetiology of depression, as well as the influence of effective therapeutic targets $[52,53]$. Moreover, our findings from the forced swim test and open field test against the control fluoxetine clearly show the CNS activity of ivacaftor at the clinically relevant dose of $40 \mathrm{mg} \cdot \mathrm{kg}^{-1}$ i.p. for 21 days (figure 3). Interestingly, non-CF mice treated with ivacaftor also displayed increased climbing/swimming activity, which could be translated into the mice having higher activity levels and correlates with the higher well-being scores seen in the AweScoreCF in patients treated with ivacaftor-lumacaftor. In addition, mice treated with the anaesthetic, psychostimulant and fast-acting antidepressant ketamine $\left(10 \mathrm{mg} \cdot \mathrm{kg}^{-1}\right.$ i.p. once) showed reduced immobility $(39.2 \pm 10.5 \mathrm{~s})$ and increased swimming/climbing activity $(200.8 \pm 8.1 \mathrm{~s})$, comparable to what was observed in the ivacaftor treatment group (figure $3 \mathrm{~b}$ and $\mathrm{c}$ ). Evidence suggests that $\mathrm{N}$-methyl-D-aspartate receptor antagonists such as ketamine could be efficacious in treating depression and anxiety [54-56]. In addition to its $\mathrm{N}$-methyl-D-aspartate receptor antagonism, ketamine possesses various other potential targets, such as effects on metabolism and transport of serotonin, noradrenaline and dopamine [57]. In the open field test, mice treated with ivacaftor showed higher locomotor activity than the fluoxetine group measured both as the number of paw touches and the total distance travelled. This is consistent with the theory of the higher well-being scores under ivacaftor therapy (figure $3 \mathrm{~d}$ and e).

Our data suggest that ivacaftor and iva-M6 also bind to both M3 and $\beta_{3}$ receptors (supplementary tables S1 and S3, and supplementary figures S7-S9). When the effects of ivacaftor and iva-M6 were tested on muscarinic receptors in the ex vivo rat colon preparation, no significant effects on colonic contractions were observed. Interestingly, when the colon was pre-treated with the $\beta_{3}$ receptor selective antagonist SR59230A, ivacaftor and iva-M6 evoked a significant contractile response in the colon. The literature suggests that $\beta_{3}$ receptor agonists are involved in smooth muscle relaxation in the colon and therefore may be involved in the inhibition of the contractile activity evoked by muscarinic receptor agonists [58]; it would appear that ivacaftor and iva-M6 could potentially simultaneously bind to both muscarinic and $\beta_{3}$ receptors in the colon. This interaction suggests complicated pharmacology as seen with ivacaftor could be acting as an allosteric modulator at the $\beta_{3}$ receptor. Further studies are currently underway to investigate this.

To confirm the findings from the in vitro binding assays and in vivo mice studies, the effect on well-being was assessed in 23 adult CF patients using the AweScoreCF questionnaire (consisting of five domains with a total of 10 questions) 1 month prior to and 3 months after ivacaftor-lumacaftor therapy (figure 4). The improvements reported were in all domains, i.e. less coughing and sputum production; improved sleep amount and quality and general health; increased levels of energy and exercise participation; increased appetite with ensuing weight gain; elevated mood levels and reduced anxiety. Treatment with ivacaftor-lumacaftor resulted in significant improvements in quality of life by 11.59 points out of a maximum of 100 points $(\mathrm{p}=0.0026)$. Over the analysed timeframe of 3 months, patients receiving standard ivacaftor-lumacaftor therapy experienced a global improvement in their quality of life compared with before initiating therapy (measured as each patient's own perception of general improvement in quality of life and mental health).

\section{Conclusions}

Recent evidence from different research groups indicates that ivacaftor and/or lumacaftor display off-target effects that may affect the clinical outcomes of ivacaftor single therapy or combination therapy with lumacaftor or tezacaftor. In the present study, we have investigated the receptor neuropharmacology of ivacaftor and its main metabolites, i.e. iva-M1 and iva-M6, to shed light on potential off-target effects including mental health parameters that could potentially affect the clinical efficacy of ivacaftor, ivacaftor-lumacaftor or future ivacaftor-tezacaftor combinations currently undergoing phase 3 studies (EVOLVE and EXPAND) [59]. 
We have shown that ivacaftor is a multifaceted drug with multimodal activity. Ivacaftor displayed a sustained CNS active profile, especially with affinity to $5-\mathrm{HT}_{2}$ receptor subtypes. Treatment with ivacaftor-lumacaftor resulted in significant improvements in the quality of life and increased well-being according to the AweScoreCF questionnaire. The current study supports the notion that ivacaftor could potentially offer the opportunity for CF patients suffering from depression and/or anxiety to improve quality of life and the health of individuals. Further investigations of the value of these off-target CNS activities are needed to determine their benefits for the treatment of CF patients who commonly suffer from mental health issues.

Acknowledgements: $K$ i determinations and receptor binding profiles were generously provided by the National Institute of Mental Health's Psychoactive Drug Screening Program (NIMH PDSP; contract HHSN-271-2013-00017-C). The NIMH PDSP is directed by Bryan L. Roth at the University of North Carolina at Chapel Hill (Chapel Hill, NC, USA) and Project Officer Jamie Driscoll at NIMH (Bethesda, MD, USA).

\section{References}

1 Schneider EK, Huang JX, Carbone V, et al. Drug-drug plasma protein binding interactions of ivacaftor. $J$ Mol Recognit 2015; 28: 339-348.

2 Ratjen F, Doring G. Cystic fibrosis. Lancet 2003; 361: 681-689.

3 Conway SP, Pond MN, Hamnett T, et al. Compliance with treatment in adult patients with cystic fibrosis. Thorax 1996; 51: 29-33.

4 Ramsey BW, Davies J, McElvaney NG, et al. A CFTR potentiator in patients with cystic fibrosis and the G551D mutation. N Engl J Med 2011; 365: 1663-1672.

5 Cholon DM, Esther CR, Gentzsch M. Efficacy of lumacaftor-ivacaftor for the treatment of cystic fibrosis patients homozygous for the F508del-CFTR mutation. Expert Rev Precis Med Drug Dev 2016; 1: 235-243.

6 Van Goor F, Hadida S, Grootenhuis PD, et al. Rescue of CF airway epithelial cell function in vitro by a CFTR potentiator, VX-770. Proc Natl Acad Sci USA 2009; 106: 18825-18830.

7 Center for Drug Evaluation and Research. Sponsor briefing document: ORKAMBI (lumacaftor/ivacaftor). Treatment of cystic fibrosis (CF) in patients age 12 years and older who are homozygous for the F508del mutation in the CFTR gene. Vertex Pharmaceuticals. 2015. www.accessdata.fda.gov/drugsatfda_docs/nda/2015/0206038Orig1s000PharmR. pdf Date last accessed: February 22, 2018.

8 Mall MA, Sheppard DN. Chronic ivacaftor treatment: getting F508del-CFTR into more trouble? J Cyst Fibros 2014; 13: 605-607.

9 Wainwright CE, Elborn JS, Ramsey BW, et al. Lumacaftor-ivacaftor in patients with cystic fibrosis homozygous for Phe508del CFTR. N Engl J Med 2015; 373: 220-231.

10 Reznikov LR, Abou Alaiwa MH, Dohrn CL, et al. Antibacterial properties of the CFTR potentiator ivacaftor. J Cyst Fibros 2014; 13: 515-519.

11 Schneider EK, Azad MA, Han ML, et al. An "unlikely" pair: the antimicrobial synergy of polymyxin B in combination with the cystic fibrosis transmembrane conductance regulator drugs KALYDECO and ORKAMBI. ACS Infect Dis 2016; 2: 478-488.

12 Adam RJ, Hisert KB, Dodd JD, et al. Acute administration of ivacaftor to people with cystic fibrosis and a G551D-CFTR mutation reveals smooth muscle abnormalities. JCI Insight 2016; 1: e86183.

13 Millan MJ, Gobert A, Lejeune F, et al. The novel melatonin agonist agomelatine (S20098) is an antagonist at 5 -hydroxytryptamine ${ }_{2 C}$ receptors, blockade of which enhances the activity of frontocortical dopaminergic and adrenergic pathways. J Pharmacol Exp Ther 2003; 306: 954-964.

14 Millan MJ, Marin P, Kamal M, et al. The melatonergic agonist and clinically active antidepressant, agomelatine, is a neutral antagonist at 5- $\mathrm{HT}_{2 \mathrm{C}}$ receptors. Int J Neuropsychopharmacol 2011; 14: 768-783.

15 Cryan JF, Lucki I. Antidepressant-like behavioral effects mediated by 5-hydroxytryptamine $2 \mathrm{C}$ receptors. $J$ Pharmacol Exp Ther 2000; 295: 1120-1126.

16 Thomsen WJ, Grottick AJ, Menzaghi F, et al. Lorcaserin, a novel selective human 5-hydroxytryptamine $2 \mathrm{C}$ agonist: in vitro and in vivo pharmacological characterization. J Pharmacol Exp Ther 2008; 325: 577-587.

17 Smith SR, Prosser WA, Donahue DJ, et al. Lorcaserin (APD356), a selective 5- $\mathrm{HT}_{2 \mathrm{C}}$ agonist, reduces body weight in obese men and women. Obesity 2009; 17: 494-503.

18 Smith BA, Modi AC, Quittner AL, et al. Depressive symptoms in children with cystic fibrosis and parents and its effects on adherence to airway clearance. Pediatr Pulmonol 2010; 45: 756-763.

19 Shen JH, Zhao Y, Rosenzweig-Lipson S, et al. A 6-week randomized, double-blind, placebo-controlled, comparator referenced trial of vabicaserin in acute schizophrenia. J Psychiatr Res 2014; 53: 14-22.

20 Palacios JM, Pazos A, Hoyer D. A short history of the $5-\mathrm{HT}_{2 \mathrm{C}}$ receptor: from the choroid plexus to depression, obesity and addiction treatment. Psychopharmacology 2017; 234: 1395-1418.

21 Hoyer D, Clarke DE, Fozard JR, et al. International Union of Pharmacology classification of receptors for 5-hydroxytryptamine (serotonin). Pharmacol Rev 1994; 46: 157-203.

22 Tecott LH, Sun LM, Akana SF, et al. Eating disorder and epilepsy in mice lacking $5-\mathrm{HT}_{2 \mathrm{C}}$ serotonin receptors. Nature 1995; 374: 542-546.

23 Dracheva S, Lyddon R, Barley $\mathrm{K}$, et al. Editing of serotonin $2 \mathrm{C}$ receptor mRNA in the prefrontal cortex characterizes high-novelty locomotor response behavioral trait. Neuropsychopharmacology 2009; 34: 2237-2251.

24 Heisler LK, Zhou L, Bajwa P, et al. Serotonin 5- $\mathrm{HT}_{2 \mathrm{C}}$ receptors regulate anxiety-like behavior. Genes Brain Behav 2007; 6: 491-496.

25 Heisler LK, Pronchuk N, Nonogaki K, et al. Serotonin activates the hypothalamic-pituitary-adrenal axis via serotonin 2C receptor stimulation. J Neurosci 2007; 27: 6956-6964.

26 Heisler LK, Tecott LH. A paradoxical locomotor response in serotonin 5- $\mathrm{HT}_{2 \mathrm{C}}$ receptor mutant mice. J Neurosci 2000; 20: RC71.

27 Millan MJ, Brocco M, Gobert A, et al. Anxiolytic properties of agomelatine, an antidepressant with melatoninergic and serotonergic properties: role of 5- $\mathrm{HT}_{2 \mathrm{C}}$ receptor blockade. Psychopharmacology 2005; 177: 448-458. 

novel therapeutic strategies. Therapie 2005; 60: 441-460.

29 Christianson JP, Ragole T, Amat J, et al. 5-hydroxytryptamine 2C receptors in the basolateral amygdala are involved in the expression of anxiety after uncontrollable traumatic stress. Biol Psychiatry 2010; 67: $339-345$.

30 Grottick AJ, Whelan K, Sanabria EK, et al. Investigating interactions between phentermine, dexfenfluramine, and 5- $\mathrm{HT}_{2 \mathrm{C}}$ agonists, on food intake in the rat. Psychopharmacology 2015; 232: 1973-1982.

31 Higgs S, Cooper AJ, Barnes NM. The 5- $\mathrm{HT}_{2} \mathrm{C}$ receptor agonist, lorcaserin, and the 5-HT $\mathrm{H}_{6}$ receptor antagonist, SB-742457, promote satiety; a microstructural analysis of feeding behaviour. Psychopharmacology 2016; 233: 417-424.

32 Harvey-Lewis $\mathrm{C}$, Li Z, Higgins GA, et al. The 5- $\mathrm{HT}_{2 \mathrm{C}}$ receptor agonist lorcaserin reduces cocaine self-administration, reinstatement of cocaine-seeking and cocaine induced locomotor activity. Neuropharmacology 2016; 101: 237-245.

33 De Luca V, Muller DJ, Hwang R, et al. $\mathrm{HTR}_{2 \mathrm{C}}$ haplotypes and antipsychotics-induced weight gain: X-linked multimarker analysis. Hum Psychopharmacol 2007; 22: 463-467.

34 Templeman LA, Reynolds GP, Arranz B, et al. Polymorphisms of the 5- $\mathrm{HT}_{2 \mathrm{C}}$ receptor and leptin genes are associated with antipsychotic drug-induced weight gain in Caucasian subjects with a first-episode psychosis Pharmacogenet Genomics 2005; 15: 195-200.

35 Martin CB, Hamon M, Lanfumey L, et al. Controversies on the role of $5-\mathrm{HT}_{2 \mathrm{C}}$ receptors in the mechanisms of action of antidepressant drugs. Neurosci Biobehav Rev 2014; 42: 208-223.

36 Di Giovanni G, Di Matteo V, Di Mascio M, et al. Preferential modulation of mesolimbic vs. nigrostriatal dopaminergic function by serotonin ${ }_{2 \mathrm{C} / 2 \mathrm{~B}}$ receptor agonists: a combined in vivo electrophysiological and microdialysis study. Synapse 2000; 35: 53-61.

37 Cathala A, Devroye C, Maitre M, et al. Serotonin $2 \mathrm{C}$ receptors modulate dopamine transmission in the nucleus accumbens independently of dopamine release: behavioral, neurochemical and molecular studies with cocaine. Addict Biol 2015; 20: 445-457.

38 Dulawa SC, Holick KA, Gundersen B, et al. Effects of chronic fluoxetine in animal models of anxiety and depression. Neuropsychopharmacology 2004; 29: 1321-1330.

39 Roth BL. National Institute of Mental Health Psychoactive Drug Screening Program Assay Protocol Book Version II. Chapel Hill, University of North Carolina, 2013.

40 Porsolt RD, Bertin A, Jalfre M. Behavioral despair in mice: a primary screening test for antidepressants. Arch Int Pharmacodyn Ther 1977; 229: 327-336.

41 Button BM, Edgeworth D, Finlayson F, et al. Effect of ivacaftor on wellness, quality of life and cognitive function in adults with cystic fibrosis and G551D mutation. J Cystic Fibros 2015; 14: Suppl., S18.

42 Porsolt RD, Le Pichon M, Jalfre M. Depression: a new animal model sensitive to antidepressant treatments. Nature 1977; 266: 730-732.

43 Cryan JF, Markou A, Lucki I. Assessing antidepressant activity in rodents: recent developments and future needs Trends Pharmacol Sci 2002; 23: 238-245.

44 Dulawa SC, Grandy DK, Low MJ, et al. Dopamine D4 receptor-knock-out mice exhibit reduced exploration of novel stimuli. J Neurosci 1999; 19: 9550-9556.

45 Prut L, Belzung C. The open field as a paradigm to measure the effects of drugs on anxiety-like behaviors: a review. Eur J Pharmacol 2003; 463: 3-33.

46 Belzung C, Le Pape G. Comparison of different behavioral test situations used in psychopharmacology for measurement of anxiety. Physiol Behav 1994; 56: 623-628.

47 Cholon DM, Quinney NL, Fulcher ML, et al. Potentiator ivacaftor abrogates pharmacological correction of $\Delta$ F508 CFTR in cystic fibrosis. Sci Transl Med 2014; 6: $246 \mathrm{ra} 296$.

48 Veit G, Avramescu RG, Perdomo D, et al. Some gating potentiators, including VX-770, diminish $\Delta$ F508-CFTR functional expression. Sci Transl Med 2014; 6: 246 ra297.

49 Matthes E, Goepp J, Carlile GW, et al. Low free drug concentration prevents inhibition of F508del CFTR functional expression by the potentiator VX-770 (ivacaftor). Br J Pharmacol 2016; 173: 459-470.

50 Lodge NJ, Li YW. Ion channels as potential targets for the treatment of depression. Curr Opin Drug Discov Devel 2008; 11: 633-641

51 Abelaira HM, Reus GZ, Quevedo J. Animal models as tools to study the pathophysiology of depression. Rev Bras Psiquiatr 2013; 35: Suppl. 2, S112-S120.

52 Can A, Dao DT, Arad M, et al. The mouse forced swim test. J Vis Exp 2012; e3638.

53 Yankelevitch-Yahav R, Franko M, Huly A, et al. The forced swim test as a model of depressive-like behavior. $J$ Vis Exp 2015; e52587.

54 Refsgaard LK, Pickering DS, Andreasen JT. Investigation of antidepressant-like and anxiolytic-like actions and cognitive and motor side effects of four $\mathrm{N}$-methyl-D-aspartate receptor antagonists in mice. Behav Pharmaco 2017; 28: 37-47

55 Berman RM, Cappiello A, Anand A, et al. Antidepressant effects of ketamine in depressed patients. Biol Psychiatry 2000; 47: 351-354.

56 Zarate CA Jr, Singh JB, Carlson PJ, et al. A randomized trial of an N-methyl-D-aspartate antagonist in treatmentresistant major depression. Arch Gen Psychiatry 2006; 63: 856-864.

57 Mion G, Villevieille T. Ketamine pharmacology: an update (pharmacodynamics and molecular aspects, recent findings). CNS Neurosci Ther 2013; 19: 370-380.

58 Guagnini F, Valenti M, Mukenge S, et al. Neural contractions in colonic strips from patients with diverticular disease: role of endocannabinoids and substance P. Gut 2006; 55: 946-953.

59 Taylor-Cousar JL, Munck A, McKone EF, et al. Tezacaftor-ivacaftor in patients with cystic fibrosis homozygous for Phe508del. N Engl J Med 2017; 377: 2013-2023. 